\title{
Akt kinase LANCL2 functions as a key driver in EGFR-mutant lung adenocarcinoma tumorigenesis
}

\author{
Yuqing Lou (1)', Jianlin Xu', Yanwei Zhang', Wei Zhang', Xueyan Zhang', Ping Gu', Hua Zhong1', Huimin Wang (1)',
} Jun Lu (B) ${ }^{1}$ and Baohui Han (i)

\begin{abstract}
Epidermal growth factor receptor (EGFR) is a key oncogene in lung adenocarcinoma (LUAD). Resistance to EGFR tyrosine kinase inhibitors is a major obstacle for EGFR-mutant LUAD patients. Our gene chip array, quantitative polymerase chain reaction validation, and shRNA-based high-content screening identified the Akt kinase lanthionine synthetase C-like protein 2 (LANCL2) as a pro-proliferative gene in the EGFR-mutant LUAD cell line PC9. Therefore, we investigated whether LANCL2 plays a role in promoting cell proliferation and drug resistance in EGFR-mutant LUAD. In silico clinical correlation analysis using the Cancer Genome Atlas Lung Adenocarcinoma dataset revealed a positive correlation between LANCL2 and EGFR expression and an inverse relationship between LANCL2 gain-of-function and survival in LUAD patients. The EGFR-mutant LUAD cell lines PC9 and HCC827 displayed higher LANCL2 expression than the non-EGFR-mutant cell line A549. In addition, LANCL2 was downregulated following gefitinib+pemetrexed combination therapy in PC9 cells. LANCL2 knockdown reduced proliferation and enhanced apoptosis in PC9, HCC827, and A549 cells in vitro and suppressed murine PC9 xenograft tumor growth in vivo. Notably, LANCL2 overexpression rescued these effects and promoted gefitinib + pemetrexed resistance in PC9 and HCC827 cells. Pathway analysis and co-immunoprecipitation followed by mass spectrometry of differentially-expressed genes in LANCL2 knockdown cells revealed enrichment of several cancer signaling pathways. In addition, Filamin A and glutathione S-transferase Mu 3 were identified as two novel protein interactors of LANCL2. In conclusion, LANCL2 promotes tumorigenic proliferation, suppresses apoptosis, and promotes gefitinib+pemetrexed resistance in EGFR-mutant LUAD cells. Based on the positive association between LANCL2, EGFR, and downstream Akt signaling, LANCL2 may be a promising new therapeutic target for EGFR-mutant LUAD.
\end{abstract}

\section{Introduction}

Lung cancer is the most prominent cause of cancer mortality worldwide, with lung adenocarcinoma (LUAD) accounting for around $50 \%$ of all lung cancers ${ }^{1}$. Mutations within the epidermal growth factor receptor (EGFR) gene are a major cause of $\operatorname{LUAD}^{2,3}$. Clinical trial evidence suggests that EGFR-mutant LUAD patients can be effectively treated using first-line EGFR tyrosine kinase

Correspondence: Huimin Wang (chestwhm@126.com) or

Jun Lu (lujun512@yahoo.com) or Baohui Han (18930858216@163.com)

'Department of Respiratory Medicine, Shanghai Chest Hospital, Shanghai Jiao Tong University, Shanghai, China

These authors contributed equally: Yuqing Lou, Jianlin Xu, Yanwei Zhang, Wei Zhang

Edited by S. Tait 
EGFR hyperactivates the serine/threonine protein kinase $A k t^{10-16}$, which is known to play a key role in supporting cell survival, proliferation, and glucose metabolism ${ }^{17}$. Akt hyperactivity is a common hallmark of a variety of human cancers, including EGFR-mutant LUAD, making it an important therapeutic target for cancer treatment ${ }^{18}$. Akt activity is subject to post-translational regulation and researchers have identified more than 20 of these regulatory proteins over the past two decades, including inhibitors and activators of Akt activity ${ }^{19}$. Notably, the enzyme lanthionine synthetase C-like 2 (LANCL2) has been identified as a novel activator of $\mathrm{Akt}^{20}$. LANCL2 interacts with both inactive Akt and the Akt kinase mTOR Complex 2 to directly promote Akt phosphorylation and cell survival in response to mitogenic signals in human liver cells ${ }^{20}$. However, the role of LANCL2 in EGFR-mutant LUAD tumorigenesis and EGFR TKI resistance (if any) has not yet been evaluated.

Therefore, we determined the correlation between LANCL2 and EGFR, and whether LANCL2 gain-offunction is associated with poor survival in LUAD patients. Furthermore, we evaluated the role of LANCL2 in cell proliferation and drug resistance in EGFR-mutant LUAD using in vitro and shRNA-mediated LANCL2 knockdown studies. In vivo tumorigenic activity was measured using a well-established murine xenograft model. Our data will provide novel insights into EGFRmutant LUAD cell proliferation.

\section{Methods}

\section{Animal welfare statement}

All animal procedures followed international, national, and institutional guidelines for humane animal treatment and complied with relevant legislation. Male BALB/c nude mice (age range: 8 - to 10-weeks old; weight range: $17-22 \mathrm{~g}$ ) were obtained from our institution's Experimental Animal Center and were housed under specific pathogen free $[\mathrm{SPF}]$, room temperature conditions with a 12-h/12-h light/dark cycle ( $n=3$ mice per cage). Mice were provided standard rodent chow (Laboratory Rodent Diet 5058; PMI Nutrition International) and sterilized tap water ad libitum. Animal health and welfare was monitored by visual inspection of general appearance, behavior, food intake, fecal output, and body weight every three days. Isoflurane was employed as a procedural anesthetic. Mice were humanely sacrificed by cervical dislocation under isoflurane anesthesia. There was no mortality events outside of planned euthanasia.

\section{In silico analysis of the Cancer Genome Atlas-Lung Adenocarcinoma (TCGA-LUAD) data}

Publicly-available data on the TCGA-LUAD cohort $(n=566)$ is retrievable from the GDC Data Portal of TCGA (https://portal.gdc.cancer.gov/). LANCL2 mRNA expression analysis, LANCL2-EGFR correlation analysis, and Kaplan-Meier survival analyses were performed on the whole TCGA-LUAD cohort using the cBioPortal for Cancer Genomics ${ }^{21}$ and the UALCAN portal ${ }^{22}$.

\section{Cell lines}

The gefitinib-sensitive human EGFR-mutant LUAD cell lines PC9 (EGFR p.Glu746_Ala750del) and HCC827 (EGFR p.Glu746_Ala750del) ${ }^{23,24}$ as well as the nonEGFR-mutant LUAD cell line A549 were purchased from American Type Culture Collection. All cells were cultured in RPMI media (Thermo Fisher) and supplemented with fetal bovine serum (10\%) and penicillin/streptomycin $(100 \mathrm{U} / \mathrm{ml})$ and cultured in a humidified environment at $37^{\circ} \mathrm{C}$ with $5 \% \mathrm{CO}_{2}$. Cell lines were tested negative for mycoplasma.

\section{Plasmid constructs and lentiviral delivery}

The lentiviral GV115 plasmid (hU6-MCS-CMV-EGFP) used all shRNA-mediated knockout experiments was acquired from GeneChem (Shanghai, China). The RNAi Consortium provided the short hairpin RNA (shRNA) sequences against all target genes. An empty GV115 plasmid was used to construct the negative control shCtrl. In order to ensure efficient gene knockdown, three independent shRNA sequences were designed for each target gene and the three separate plasmids carrying the three unique shRNA sequences were mixed in equal proportion during GV115 lentiviral packaging. Cells were cultured until they reached 50\% confluency and were then incubated with GV115 lentiviral particles and polybrene $(4 \mu \mathrm{g} / \mathrm{mL})$.

The lentiviral GV358 plasmids (Ubi-MCS-3FLAGSV40-EGFP-IRES-puromycin) used for LANCL2 overexpression was also acquired from GeneChem. For LANCL2 overexpression, a synonymous LANCL2 mutant was constructed in which the shLANCL2 binding sequence was mutated from GGAAGATCATTCATAATTT to GcAAaATtATcCAcAAcTT.

\section{Gene chip arrays}

Two whole human cDNA gene chip arrays were performed here using a similar methodology: (i) untreated PC9 cells to determine the most abundant genes in PC9 cells, and (ii) shLANCL2-infected PC9 cells vs. shCtrlinfected PC9 cells to determine differentially-regulated genes arising from LANCL2 knockdown in PC9 cells.

Briefly, the RNeasy Plus Mini Kit (Qiagen) was used to extract RNA. RNA was transcribed into cDNA and the cDNA product was amplified using an OneArray plus RNA kit (Phalanx Biotech Group). The Human Whole Genome OneArray was incubated with Cy5-labeled RNA targets to allow for hybridization. The signal intensity was quantified via the Agilent Microarray Scanner (Agilent). 
Data transformation and normalization were performed with GeneSpring software. Standardization of the data was performed using the RMA algorithm.

For the shLANCL2 vs. shCtrl PC9 cell analysis, significant differences between groups were identified using a non-paired $t$-test corrected by the Benjamini-Hochberg method. A corrected $p$ value (i.e., FDR) threshold of $\leq 0.05$ and a fold-change threshold of greater than or equal to \pm 2.0 were applied to identify the differentially-regulated probes. The differentially-regulated genes were obtained therefrom using the priority rule of the probe set. The Heatmap. 2 function from the gplots package in R Project was used for clustering analysis of the differentiallyregulated genes. The Euclidean matrix distance calculation and complete linkage methods were employed to perform the clustering analysis.

In the untreated PC9 cell analysis, the top 32 most abundant genes were selected for quantitative RT-PCR (qPCR) validation. In the shLANCL2 vs. shCtrl PC9 cell analysis, all downstream genes identified by pathway analysis were selected for qPCR validation.

\section{Gefitinib + pemetrexed combination therapy}

Where indicated, PC9 cells and HCC827 cells were subjected to 72-h exposure of PBS vehicle control or $10 \mathrm{nM}$ gefitinib $\left(\mathrm{IC}_{50}: 13 \mathrm{nM}\right.$ and $8 \mathrm{nM}$, respectively) plus $20 \mathrm{nM}$ pemetrexed $\left(\mathrm{IC}_{50}: 50 \mathrm{nM}\right.$ and $500 \mathrm{nM}$, respectively) ${ }^{23}$.

\section{Quantitative RT-PCR (qPCR)}

TRIzol (Shanghai Pufel) was used to extract RNA, and the concentration and quality of total RNA were measured by Nanodrop spectrophotometer. Reverse transcription was performed according to the kit instructions (Promega M-MLV kit). The qPCR protocol was as follows: $95^{\circ} \mathrm{C}$ for $30 \mathrm{~s} ; 95^{\circ} \mathrm{C}$ for $5 \mathrm{~s}$ and $60^{\circ} \mathrm{C}$ for $30 \mathrm{~s}$ for a total of 45 cycles. The qPCR reaction reagent was SYBR Master Mixture (TAKARA, DRR041B0). The LANCL2 primers were $\left(5^{\prime}\right.$ to $\left.3^{\prime}\right)$ : forward-GTG TAG CGA TGT GAT TTG GC and reverse-AAT GCT GGA AAC CGT GAT GT. The GAPDH primers were $\left(5^{\prime}\right.$ to $\left.3^{\prime}\right)$ : forwardTGA CTT CAA CAG CGA CAC CCA and reverse-CAC CCT GTT GCT GTA GCC AAA. All other primers were designed and synthesized by Sheng Gong Co. Ltd. (Shanghai, China). GAPDH housekeeping gene expression was used to normalize target gene expression and

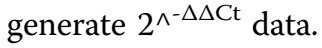

\section{High content screening (HCS) via lentiviral gene knockdown}

Twenty-four hours after lentiviral infection (day 0), a HCS assay was performed in the infected PC9 cells by incubating them for 4 days (days 1-4) to assess cell proliferation. Cell counts were measured by fluorescence imaging microscopy every day. Quantitative results were analyzed with ArrayScan HCS software (Cellomics, Inc., Pittsburgh, PA, USA). To verify the results of the HCS, the top three gene targets, which produced the greatest suppression in PC9 cell proliferation, were selected for functional verification using individual shRNAs. qPCR was performed to verify efficient gene knockdown for each individual shRNA.

\section{CCK-8 assay}

The CCK- 8 assay was used to assess cell proliferation. In brief, 2000 cells per well were plated into a 96-well plate with $150 \mu \mathrm{L}$ of media and incubated for 24 hours at $37^{\circ} \mathrm{C}$. Next, $10 \mu \mathrm{L}$ of the CCK-8 solution (Sigma, 96992) was added for the final $4 \mathrm{~h}$ of the day prior to oscillation for $5 \mathrm{~min}$. Absorbance values $(450 \mathrm{~nm}$, reference wavelength of $650 \mathrm{~nm}$ ) were obtained on a microplate reader (M2009PR, Tecan Infinite). This was repeated for five days.

\section{Flow cytometric detection of cell apoptosis}

Annexin V-APC (eBioscience, 88-8007) was used to evaluate cell apoptosis via flow cytometry. Annexin V monochromatic staining was used here since the lentiviral-infected cells were already labeled with GFP. Briefly, PC9 cells $\left(5 \times 10^{5}\right)$ were plated in six-well plates. Trypsin (Gibco) was used to detach cells, then cells were washed with PBS for a total of three times before being resuspended in $1 \times$ binding buffer $(200 \mu \mathrm{l})$ and incubated with Annexin V-APC at room temperature for $15 \mathrm{~min}$ in the dark. After adjusting $1 \times$ binding buffer volume, cells were analyzed on a BD FACS Aria II (BD Biosciences).

\section{Caspase-3/7 activity assay}

The Caspase-Glo 3/7Assay Kit (Promega, G8091) was used to assay caspase-3/7 activity according to the kit instructions. Absorbance values $(485 \mathrm{~nm}$ excitation, $520 \mathrm{~nm}$ emission) were obtained on a microplate reader (M2009PR, Tecan Infinite).

\section{Western blotting}

Cells were lysed using pre-cooled $2 \times$ Lysis Buffer $(1 \mathrm{M}$ Tris- $\mathrm{HCl}$ (pH 6.8), 2\% $100 \mathrm{mM}$ mercaptoethanol, 20\% glycerin, and 4\% SDS) for $15 \mathrm{~min}$ followed by ultrasound ( $200 \mathrm{~W}$, four 5 -s cycles, $2 \mathrm{~s}$ between cycles). The BCA protein assay was performed according to the kit instructions (Beyotime) to standardize each sample to a $2 \mu \mathrm{g} / \mu \mathrm{L}$ protein concentration. Lysates were then loaded, ran and separated on polyacrylamide gels. The protein was then transferred from the gel and onto PVDF membranes. Membranes were incubated at $4{ }^{\circ} \mathrm{C}$ overnight with the following primary antibodies (diluted in TBST solution with $5 \%$ skim milk as indicated): antiLANCL2 (1:500, ab88860), anti-FLNA (1:1000, 
ab76289), anti-FASN (1:1000, ab128870), anti-TRIM25 (1:1000, ab167154), anti-YWHAB (1:1000, ab32560) (all from Abcam); anti-GSTM3 (1:1000, 15214-1-AP), antiPKLR (1:1000, 22456-1-AP), anti-YWHAE (1:1000, 11648-2-AP) (all from Proteintech); and anti-GAPDH (1:2000, sc-32233, Santa Cruz Biotechnology). The following day, membranes were incubated for $1.5 \mathrm{~h}$ at room temperature with secondary horseradish peroxidase-conjugated goat anti-mouse IgG (1:5000, sc2005, Santa Cruz Biotechnology) diluted in TBST solution with $5 \%$ skim milk. Finally, the Pierce ECL Western Blotting Substrate (Thermo) and autoradiography were used to detect and visualized proteins on the membrane and protein levels were quantified using the software Quantity One 4.6

\section{Nude murine xenograft model}

The BALB/c nude mice $(n=30)$ were acclimatized for one week prior to PC9 cell injection. Animals were randomly segregated into the three experimental cohorts ( $n=10$ mice per cohort) using a random number generator. Lentivirus-infected PC9 cells $\left(1 \times 10^{7}\right)$ diluted in $200 \mu \mathrm{l}$ Matrigel (1:1 in culture medium, Corning Life Sciences) were subcutaneously injected into the right flanks of anesthetized nude mice. Sample size was determined based on previous studies using similar xenograft tumor models ${ }^{25,26}$. Xenograft tumors volumes were measured every week and monitored for a total of six weeks (the predetermined endpoint). Following humane sacrifice, the xenograft tumors were dissected out, weighed, and measured. Tumor volumes were calculated as follows: volume $=0.5 \times$ length $\times$ width $^{2}$.

\section{Co-immunoprecipitation/mass spectrometry (Co-IP/MS) analysis}

For the Co-IP experiment, PC9 cells were infected with a $3 \times$ FLAG-labeled LANCL2-overexpressing GV491 lentivirus or a negative control GV491 lentivirus. After puromycin selection, anti-FLAG M2 Affinity Gel Magnetic Beads (A2220, Sigma, St. Louis, MO, USA) were used to capture the fusion proteins according to the manufacturer's instructions. The immunoprecipitates were washed with lysis buffer and boiled in sample loading buffer for immunoblotting analysis.

For the MS experiment, proteins were resolved on a 10\% SDS-PAGE and stained with Coomassie Brilliant Blue (R250). The excised stained gel pieces were acetonitrile-dehydrated, vacuum-dried, and trypsin-digested. The trypsin-digested peptides were recovered, dried, and resuspended in 50\% CAN and 0.1\% TFA. The peptide mixture was subjected to nano-liquid chromatographytandem MS (LC-MS/MS) with a LTQ Velos-Orbitrap MS (Thermo Scientific, Waltham, MA, USA) coupled to an Ultimate RSLC nano-LC system (Dionex).

\section{Pathway and functional enrichment analysis}

We used ClueGO Cytoscape plugin ${ }^{27}$, Ingenuity Pathways Analysis (IPA, Ingenuity Systems, Inc., Redwood City, CA, www.ingenuity.com) $)^{28}$, and STRING ${\mathrm{v} 11^{29}}^{29}$ bioinformatics tools to identify significantly enriched pathways, biological functions and disease, as well as functional relationships between genes and gene networks.

\section{Statistics}

Data are reported as means \pm standard deviations unless otherwise specified. All data was statistically analyzed using Student's $t$-test (two-tailed) or one-way ANOVA with Bonferroni's multiple comparison test. A $p$ value of $<$ 0.05 was deemed significant for all analyses.

\section{Results}

LANCL2 is a key gene contributing to PC9 cell proliferation

We conducted a transcriptomic screen employing a gene chip array and follow-up qPCR validation to identify the most abundant gene transcripts in the PC9 LUAD cell line. qPCR analysis of the top 32 most-abundant gene transcripts identified from the gene chip array revealed several highly-abundant genes, including RPS4X, EIF3C, DHX9, TMEM123, and LANCL2 (Supplementary Fig. S1). Thereafter, we assessed the roles of these highlyabundant genes in PC9 cell proliferation using high content screening (HCS). On day 0, genes in PC9 cells were individually silenced using lentiviral shRNAmediated knockdown, followed by cell count analysis conducted from day 1 to day 4 . We found that certain shRNAs significantly suppressed PC9 cell proliferation over the four-day period (Fig. 1a and b). Specifically, the shRNAs targeting RPS4X, EIF3C, and LANCL2 displayed the most profound anti-proliferative effects; therefore, these shRNAs were selected for individual verification. After verification using HCS, we discovered that shLANCL2 more potently suppressed PC9 proliferation in vitro relative to shRPS4X or shEIF3C (Fig. 1c and d). This evidence suggested that LANCL2 plays a role in promoting PC9 cell proliferation; therefore, we selected LANCL2 as a target gene for further study.

\section{LANCL2 is positively correlated with EGFR in LUAD patients; high LANCL2 expression is associated with poor survival}

We analyzed LANCL2 expression in the TCGA-LUAD cohort $(n=566)$ to ascertain the clinical relevance of LANCL2. No significant differences in LANCL2 mRNA expression were observed between LUAD tumors and normal lung tissue (Supplementary Fig. S2a). However, after screening the top 25 gene candidates with the highest expressional correlations with LANCL2, we identified the LUAD oncogene EGFR (Supplementary Fig. 


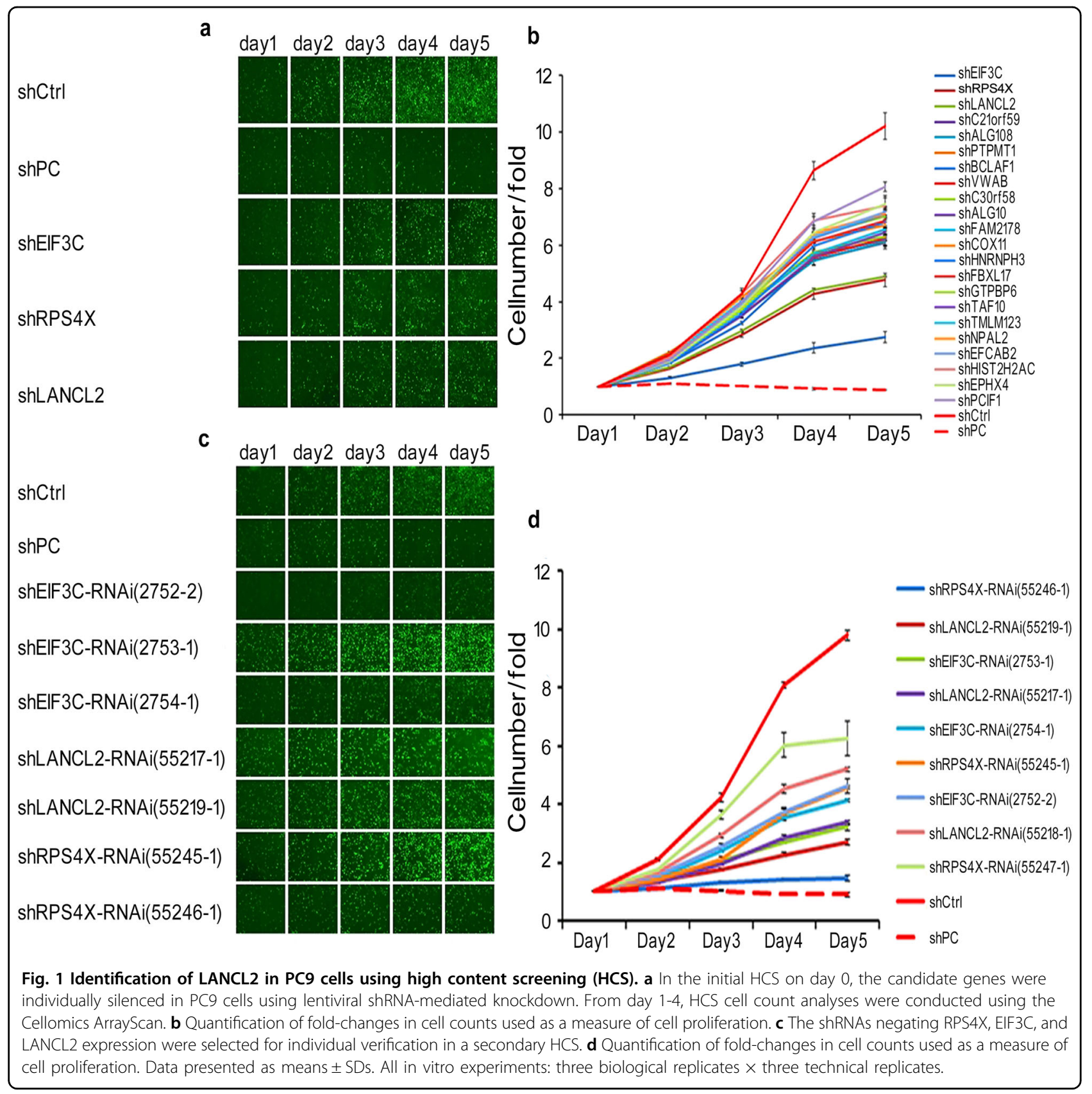

S2b). Notably, EGFR was the only one of these 25 candidate genes that possessed a significant mutational cooccurrence with LANCL2 (Supplementary Fig. S2c). Moreover, LUAD tumor LANCL2 mRNA expression and LUAD tumor EGFR mRNA expression displayed a strong positive correlation (Pearson $r=0.63$; Supplementary Fig. S2d). In addition, Kaplan-Meier survival analyses, using LANCL2 mutational status (Supplementary Fig. S2e) and LANCL2 mRNA expression (Supplementary Fig. S2f), revealed that LANCL2 gain-of-function is significantly associated with inferior overall survival outcomes for LUAD patients. This combined evidence indicates that
LANCL2 and EGFR expression are positively correlated, and high LANCL2 expression is associated with poor survival, in LUAD patients.

\section{LANCL2 knockdown suppresses EGFR-mutant LUAD cell proliferation}

We assayed three LUAD cell lines to determine their relative expression of LANCL2 and detected that the EGFR-mutant cell lines, namely PC9 and HCC827, displayed higher LANCL2 expression than the non-EGFRmutant cell line A549 (Fig. 2a). These in vitro findings mirror the positive correlation observed between LANCL2 


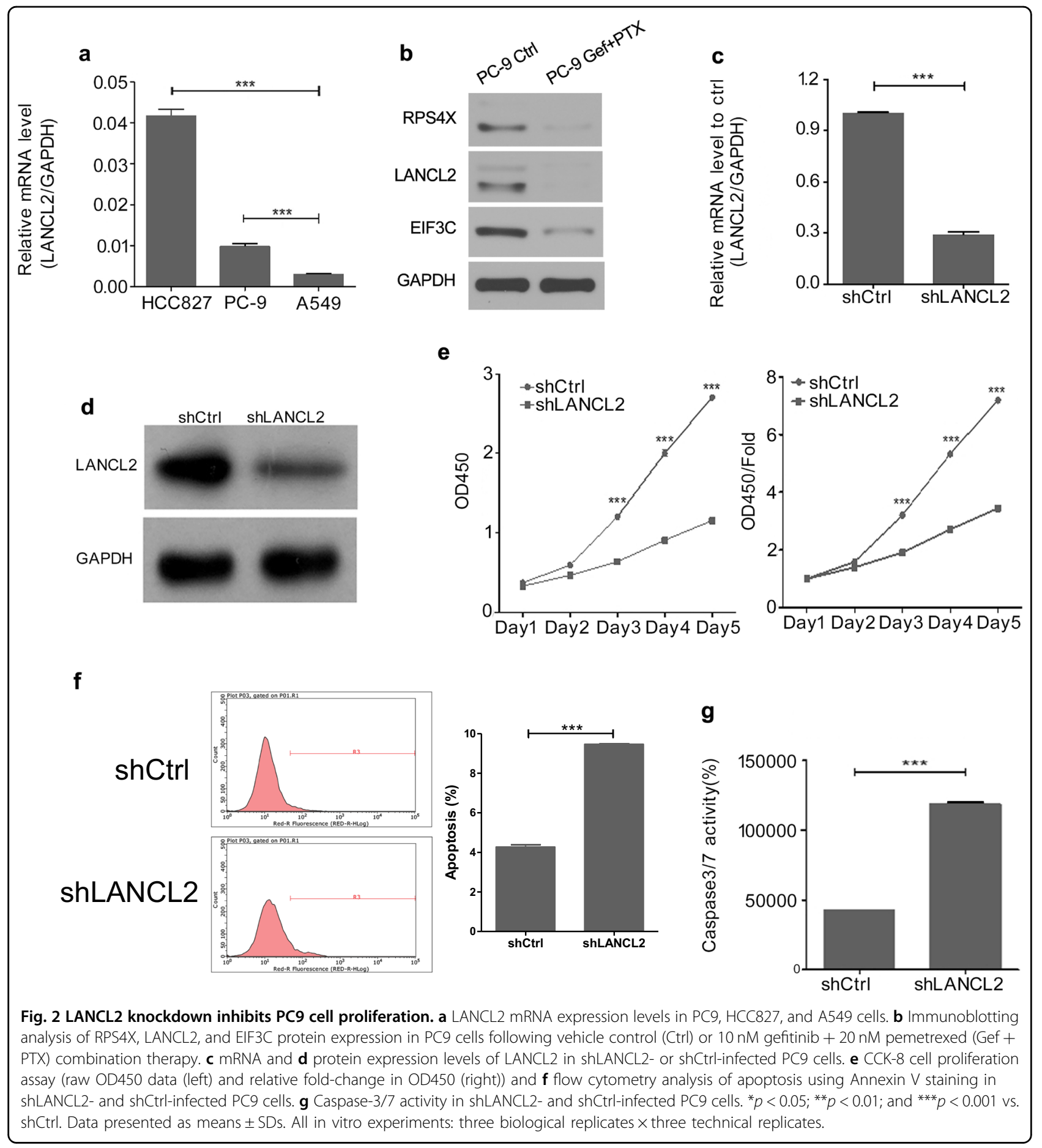

mRNA expression and EGFR mRNA expression in LUAD tumors (Supplementary Fig. S2). To assess the effects of gefitinib + pemetrexed combination therapy on the three key genes identified in the HCS (i.e., LANCL2, RPS4X, and EIF3C), we treated PC9 cells with vehicle control or $10 \mathrm{nM}$ gefitinib $+20 \mathrm{nM}$ pemetrexed (Gef + PTX). Immunoblotting analysis revealed that LANCL2, as well as RPS4X and EIF3C, were significantly downregulated following Gef +
PTX combination therapy (Fig. 2b). This combined data suggest that LANCL2 may play a role in cell proliferation and drug resistance in EGFR-mutant LUAD cells.

To further investigate the role of LANCL2 in EGFRmutant LUAD cells, PC9 cells were transfected with a shRNA against LANCL2 (55219-1, hereinafter referred to as shLANCL2) to knockdown LANCL2 expression. Both mRNA and protein expression levels of LANCL2 were 
a

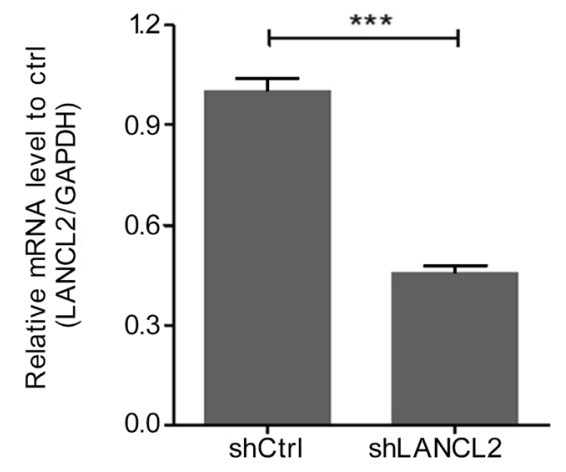

C

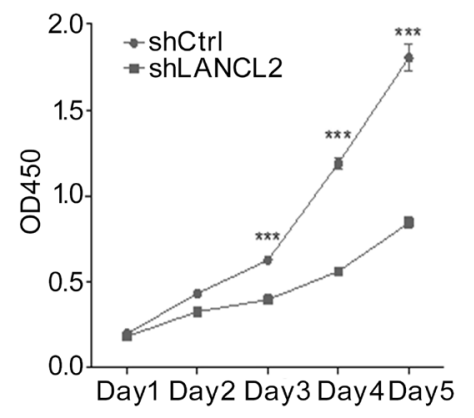

b

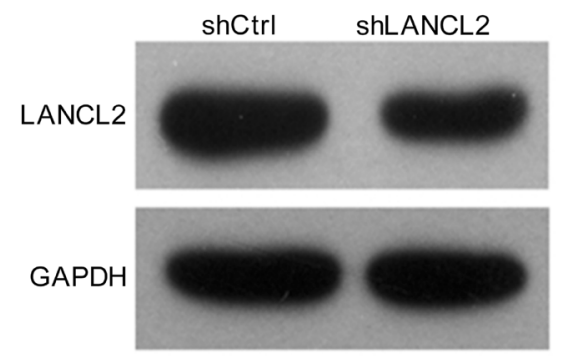

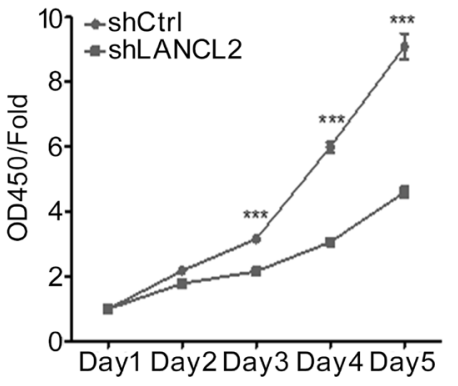

d

e
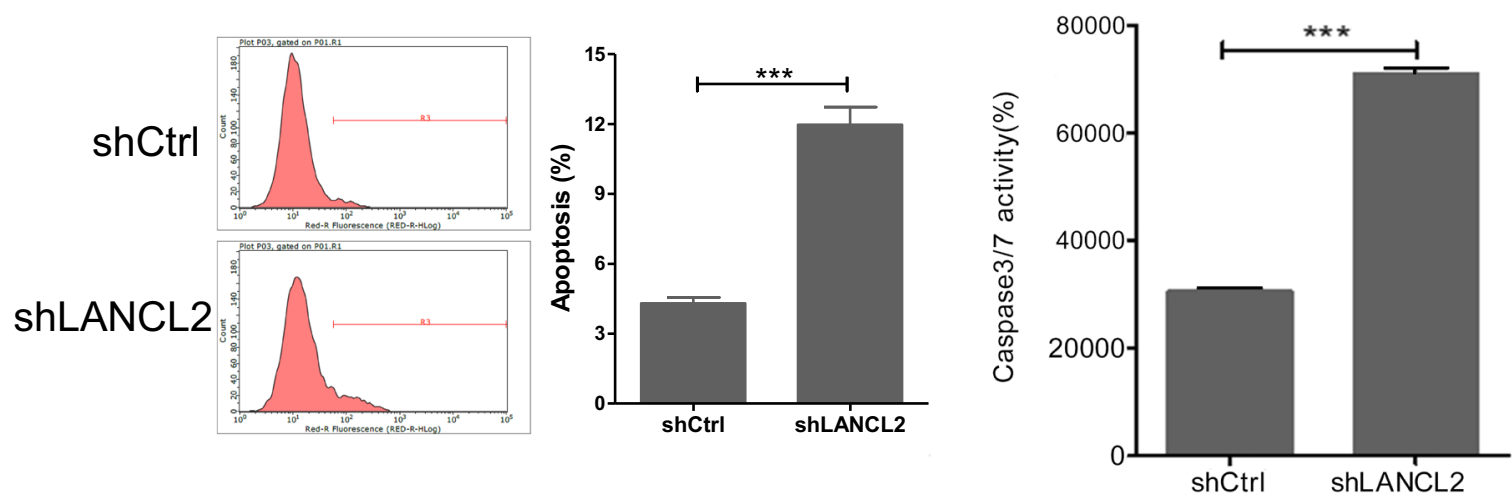

Fig. 3 LANCL2 knockdown inhibits HCC827 cell proliferation. a mRNA and $\mathbf{b}$ protein expression levels of LANCL2 in shLANCL2- or shCtrl-infected HCC827 cells. c CCK-8 cell proliferation assay (raw OD450 data (left) and relative fold-change in OD450 (right)) and $\mathbf{d}$ flow cytometry analyses of apoptosis using Annexin V staining in shLANCL2- and shCtrl-infected HCC827 cells. e Caspase-3/7 activity in shLANCL2- and shCtrl-infected HCC827 cells. ${ }^{*} p<0.05$; ${ }^{* *} p<0.01$; and ${ }^{* * *} p<0.001$ vs. shCtrl. Data presented as means \pm SDs. All in vitro experiments: three biological replicates $\times$ three technical replicates.

notably reduced following shLANCL2 transfection (Fig. $2 \mathrm{c}$ and $\mathrm{d})$. Additionally, the proliferation of shLANCL2transfected PC9 cells was significantly lower than that of shCtrl-transfected PC9 cells (Fig. 2e). Furthermore, shLANCL2-transfected PC9 cells demonstrated elevated levels of cell apoptosis (Fig. 2f) and cleaved caspase-3/7 activity (Fig. 2g). To confirm these findings, we repeated the foregoing experiments in the EGFR-mutant HCC827 cell line. After effective LANCL2 knockdown (Fig. 3a and b), we evidenced similar findings in HCC827 cells with respect to cell proliferation (Fig. 3c), apoptosis (Fig. 3d), and caspase-3/7 activity (Fig. 3e).

To investigate the role of LANCL2 in non-EGFRmutant LUAD cells, A549 cells were transfected with shLANCL2 to knockdown LANCL2 expression (Supplementary Fig. S3a and b). Similar to the EGFR-mutant LUAD cell lines, the proliferation of shLANCL2transfected A549 cells was significantly lower than that of shCtrl-transfected A549 cells (Supplementary Fig. S3c). Furthermore, shLANCL2-transfected A549 cells 
demonstrated elevated levels of cell apoptosis (Supplementary Fig. S3d). These combined findings suggest that LANCL2 plays a pro-proliferative role in EGFR-mutant and non-EGFR-mutant LUAD cells.

\section{LANCL2 overexpression rescues LANCL2 knockdown- induced suppression of EGFR-mutant LUAD cell proliferation}

To validate the role of LANCL2 in PC9 and HCC827 cells, we devised a set of gene recovery experiments consisting of three experimental groups: a negative control group (LANCL2-NC + OE-NC), LANCL2 knockdown group (LANCL2-KD + OE-NC), and LANCL2 knockdown with
LANCL2 rescue overexpression group (LANCL2-KD + OE). To effectively knockdown LANCL2 expression, LANCL2$\mathrm{KD}+\mathrm{OE}-\mathrm{NC}$ cells were infected with a lentiviral vector containing shLANCL2. LANCL2-KD + OE cells, infected with a lentiviral vector containing a synonymous mutant of the LANCL2 open reading frame that lacked the shLANCL2 binding site (Supplementary Fig. S4), led to effective LANCL2 overexpression in the presence of shLANCL2. Following infection, the knockdown and overexpression of LANCL2 in LANCL2-KD + OE-NC and LANCL2-KD + $\mathrm{OE}$ cells, respectively, was confirmed using $\mathrm{qPCR}$ and immunoblotting in PC9 cells (Fig. 4a and b). LANCL2-OE rescued the decreased cell proliferation produced by

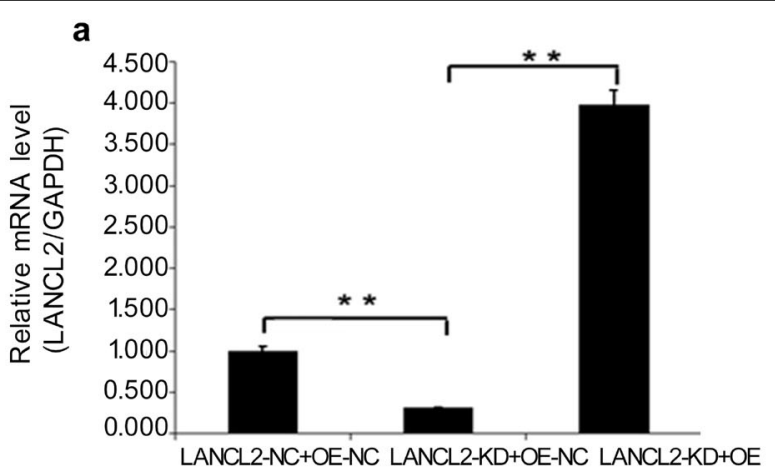

b
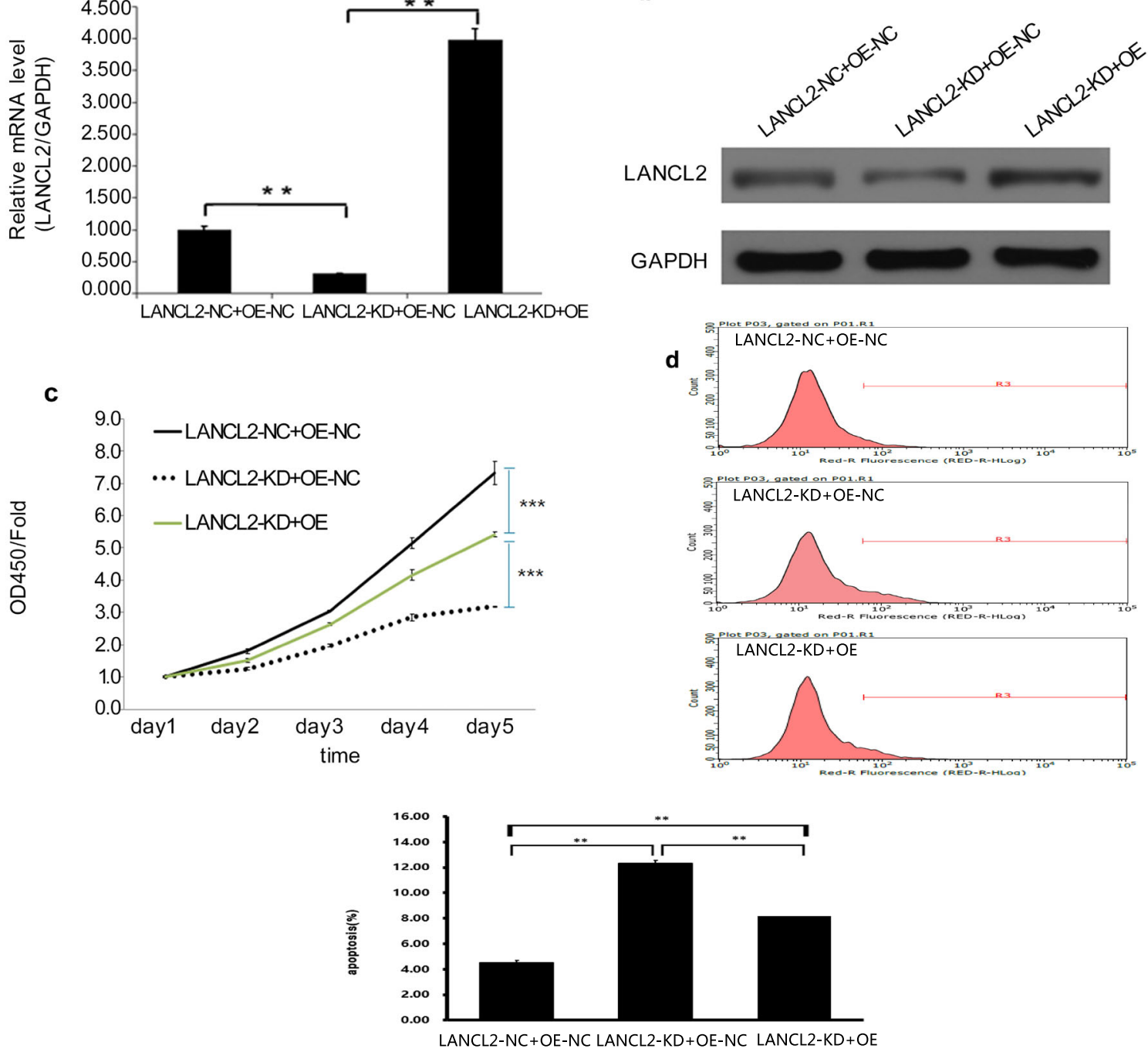

Fig. 4 LANCL2 overexpression rescues effects of LANCL2 knockdown in PC9 cells. a mRNA and $\mathbf{b}$ protein expression levels of LANCL2 in negative control (LANCL2-NC + OE-NC), LANCL2 knockdown (LANCL2-KD + OE-NC), and LANCL2 knockdown with LANCL2 rescue overexpression (LANCL2-KD + OE) PC9 cells. c CCK-8 cell proliferation assay and $\mathbf{d}$ flow cytometry analyses of apoptosis using Annexin $V$ staining in infected PC9 cells. ${ }^{*} p<0.05 ;{ }^{* *} p<0.01$; and ${ }^{* * *} p<0.001$ vs. LANCL2-NC + OE-NC. Data presented as means \pm SDs. All in vitro experiments: three biological replicates $\times$ three technical replicates. 

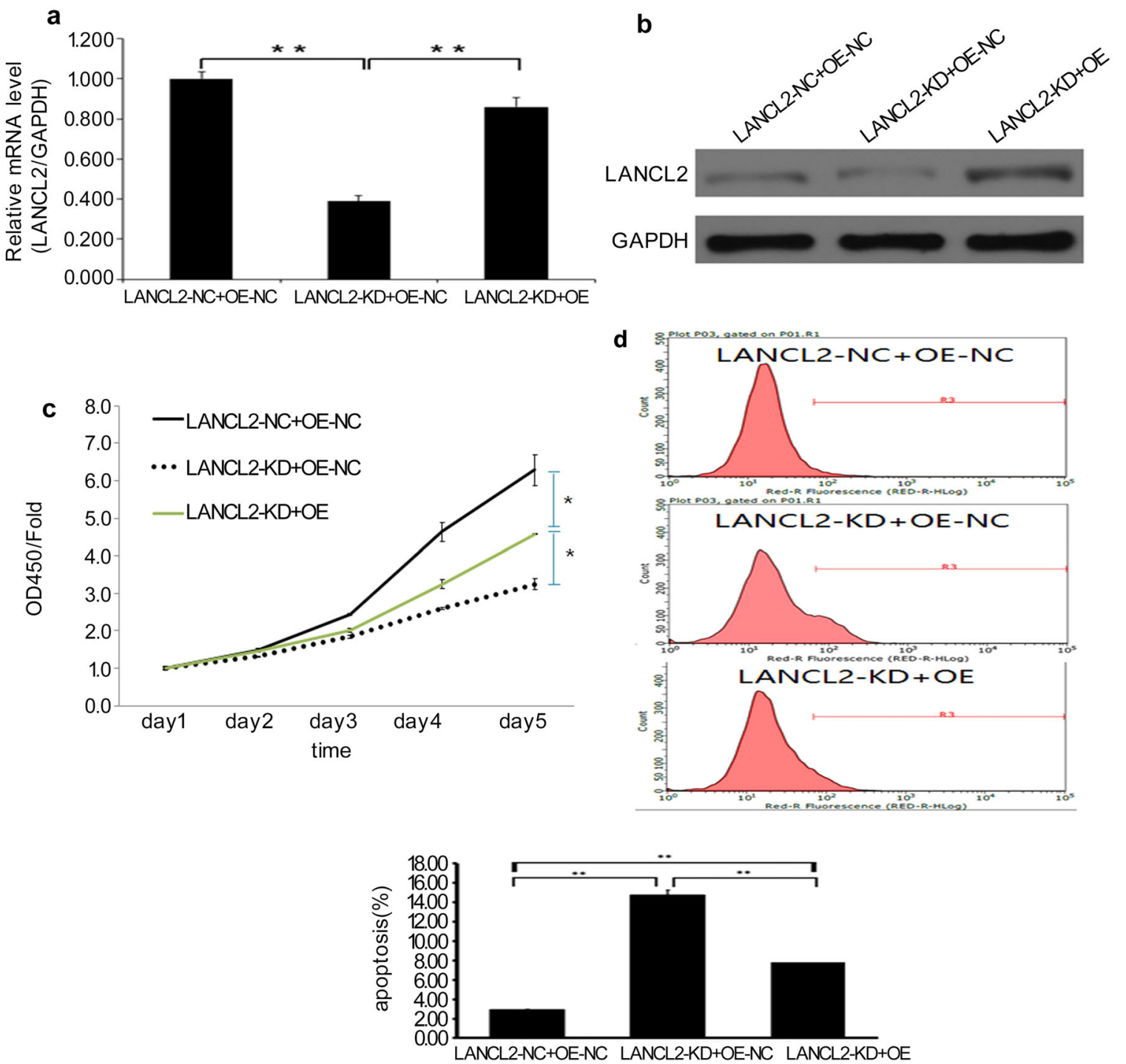

Fig. 5 LANCL2 overexpression rescues effects of LANCL2 knockdown in HCC827 cells. a mRNA and $\mathbf{b}$ protein expression levels of LANCL2 in negative control (LANCL2-NC + OE-NC), LANCL2 knockdown (LANCL2-KD + OE-NC), and LANCL2 knockdown with LANCL2 rescue overexpression (LANCL2-KD + OE) HCC827 cells. c CCK-8 cell proliferation assay and $\mathbf{d}$ flow cytometry analyses of apoptosis using Annexin $V$ staining in infected HCC827 cells. ${ }^{*} p<0.05 ;{ }^{* *} p<0.01$; and ${ }^{* * *} p<0.001$ vs. LANCL2-NC + OE-NC. Data presented as means \pm SDs. All in vitro experiments: three biological replicates $\times$ three technical replicates.

LANCL2-KD in PC9 cells (Fig. 4c). LANCL2-OE also rescued the increased apoptosis produced by LANCL2-KD in PC9 cells (Fig. 4d). To confirm these findings, we repeated the foregoing experiments in the HCC827 cell line. After effective LANCL2 knockdown and overexpression (Fig. 5a and b), we evidenced similar findings in HCC827 cells with regard to cell proliferation (Fig. 5c) and apoptosis (Fig. 5d).

\section{LANCL2 overexpression rescues LANCL2 knockdown- induced suppression of EGFR-mutant LUAD xenograft tumor growth}

A nude murine PC9 xenograft tumor model was constructed to assess whether the loss or rescue of LANCL2 expression affects the growth of EGFR-mutant LUAD xenograft tumors in vivo. Therefore, 30 nude mice were injected with PC9 cells infected with either LANCL2-NC + OE-NC $(n=10$ mice), LANCL2-KD + OE-NC $(n=10$ mice), or LANCL2-KD + OE $(n=10$ mice $)$ as described above. Visible tumors were detectable 1-week post injection. We tracked tumor growth for 6 weeks and noted LANCL2-KD + OE-NC tumors grew slower than control LANCL2-NC + OE-NC tumors, while LANCL2$\mathrm{OE}$ rescued this effect (Fig. 6a). At six weeks, the LANCL2-KD + OE-NC tumors were significantly smaller in size and weight than control LANCL2-NC + OE-NC tumors (Fig. 6a-c). LANCL2-OE rescued the decreased 


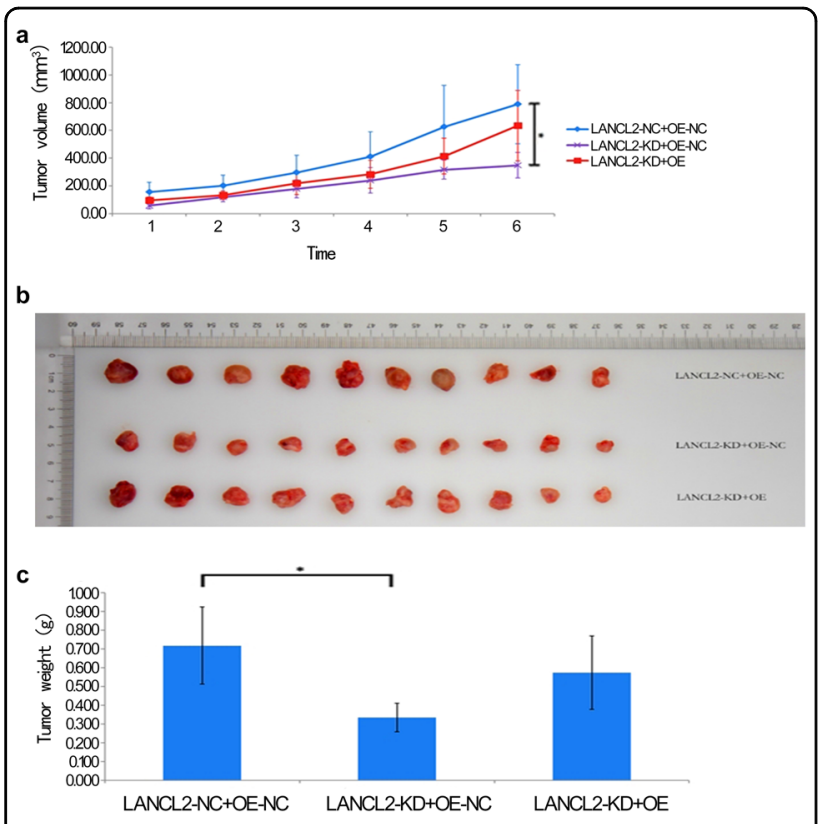

Fig. 6 LANCL2 knockdown reduces PC9 xenograft tumor growth in vivo, while LANCL2 overexpression rescues effects of LANCL2 knockdown. a PC9 xenograft tumor volumes measured over a 6week period ( $n=10$ mice per group). $\mathbf{b}$ Images of the extracted PC9 xenograft tumors after 6 weeks. c Quantification of the final PC9 xenograft tumor weights. ${ }^{*} p<0.05 ;{ }^{* *} p<0.01$; and ${ }^{* * *} p<0.001$ vs. LANCL2-NC + OE-NC. Data presented as means \pm SDs.

tumor size and weight produced by LANCL2-KD (Fig. 6a-c).

LANCL2 overexpression promotes gefitinib + pemetrexed resistance in EGFR-mutant LUAD cells

To assess whether LANCL2 expression affects gefitinib + pemetrexed resistance in EGFR-mutant LUAD cells, we devised a set of LANCL2 overexpression experiments in treated PC9 and HCC827 cells using the following groups: a gefitinib-treated negative control group (Vector + Gefitinib), gefitinib+pemetrexed-treated negative control group (Vector + Gefitinib\&Pemetrexed), and gefitinib + pemetrexed-treated LANCL2 overexpression group (LANCL2 + Gefitinib\&Pemetrexed). Cells were treated with $10 \mathrm{nM}$ gefitinib and $20 \mathrm{nM}$ pemetrexed as indicated. Following infection, the overexpression of LANCL2 in PC9 cells was confirmed using $\mathrm{QPCR}$ and immunoblotting (Supplementary Fig. S5a and b). LANCL2 overexpression abrogated the suppressive effect of gefitinib+pemetrexed on PC9 cell proliferation (Supplementary Fig. S5c). In addition, LANCL2 overexpression nullified the positive effect of gefitinib+pemetrexed on PC9 cell apoptosis (Supplementary Fig. S5d) produced by LANCL2-KD. To confirm these findings, we repeated the foregoing experiments in the HCC827 cell line. After effective LANCL2 knockdown and overexpression (Supplementary
Fig. S6a and b), similar findings were obtained with respect to cell proliferation (Supplementary Fig. S6c) and apoptosis (Supplementary Fig. S6d) in HCC827 cells.

\section{LANCL2 knockdown-induced DEGs associated with cancer- related signaling pathways and proteins}

To improve understanding of the underlying molecular pathways responsible for the effects of LANCL2 on EGFRmutant LUAD cells, we conducted gene chip array analysis in PC9 cells with and without LANCL2 knockdown. We discovered that the loss of LANCL2 produced 496 upregulated and 839 downregulated DEGs, based on our selected threshold values (Fig. 7a and b). Using these DEGs as inputs for ClueGO analyses identified pathways for protein localization (GO-BP), enzyme binding (GO-MF), and endoplasmic reticulum (GO-CC) were most enriched (Supplementary Fig. S7). Additionally, the ClueGO Kyoto Encyclopedia of Genes and Genomes (KEGG)/BioCarta pathway analyses of these DEGs revealed pathways in cancer, and the PPARA and Wnt signaling pathways were most significantly enriched (Supplementary Fig. S8). IPA was used for classical pathway analysis and revealed that DEGs were significantly enriched in HER2 breast cancer, non-small cell lung cancer, and melanoma signaling pathways (Supplementary Fig. S9a), while IPA disease and biological function analysis detected significant enrichment of cancer and organismal injuries and abnormalities (Supplementary Fig. S9b). We also performed an IPA interaction network analysis (which identifies regulatory events leading from signaling events to transcriptional effects; Fig. 7c), an IPA upstream regulator analysis (which predicts relevant transcriptional regulators and whether they are likely activated or inhibited; Fig. 7d), and an IPA downstream effects analysis (which identifies biological functions expected to increase or decrease; Fig. 7e). Notably, the IPA interaction network analysis revealed putative interactions between LANCL2 and EGFR, LANCL2 and amyloid precursor protein (APP), and LANCL2 and its traditional target Akt (Fig. 7c). We pursued qPCR validation of interactors of LANCL2 from the IPA interaction network analysis and found that loss of LANCL2 in PC9 cells resulted in the downregulation of several key genes (e.g., CCND1, WNT5A, and TP53) and upregulation of several key genes (e.g., APP, EGFR, and DKK1) (Supplementary Fig. S10a). Western blotting validated that LANCL2 knockdown significantly downregulated BMPR2 and $\mathrm{NOTCH} 2$ protein expression while significantly upregulating DUSP1 protein expression (Supplementary Fig. S10b).

\section{LANCL2 directly interacts with Filamin A (FLNA) and glutathione S-transferase Mu 3 (GSTM3)}

To further explore mechanism of action of LANCL2 in EGFR-mutant LUAD cells, we screened for putative 


\section{a}
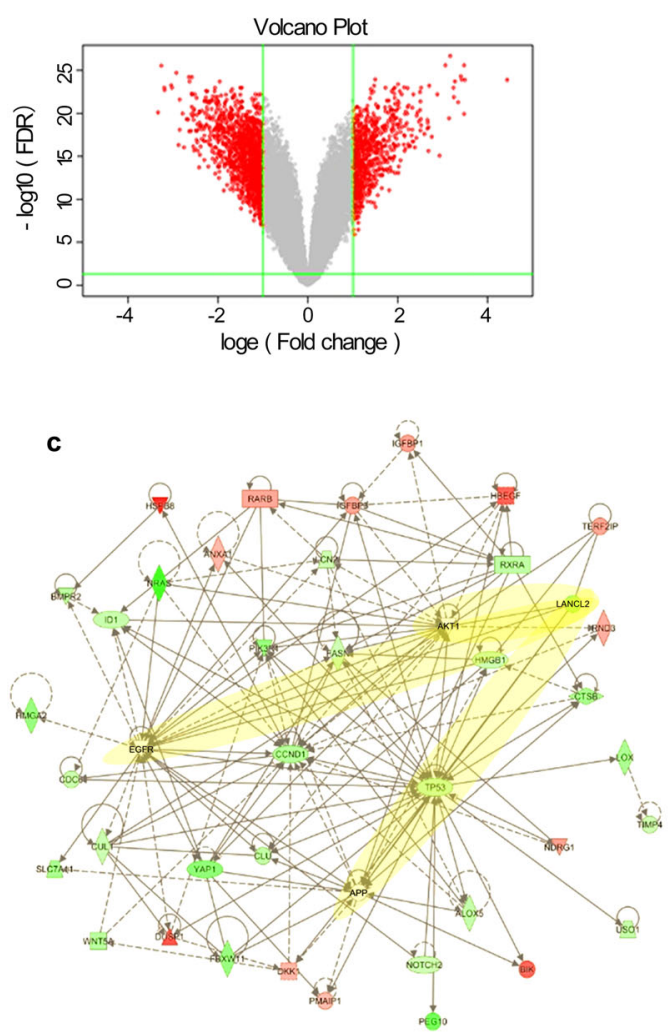

e

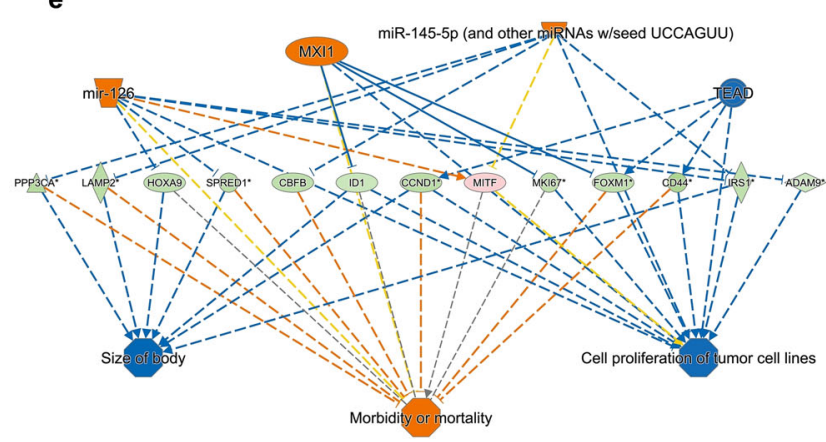

b
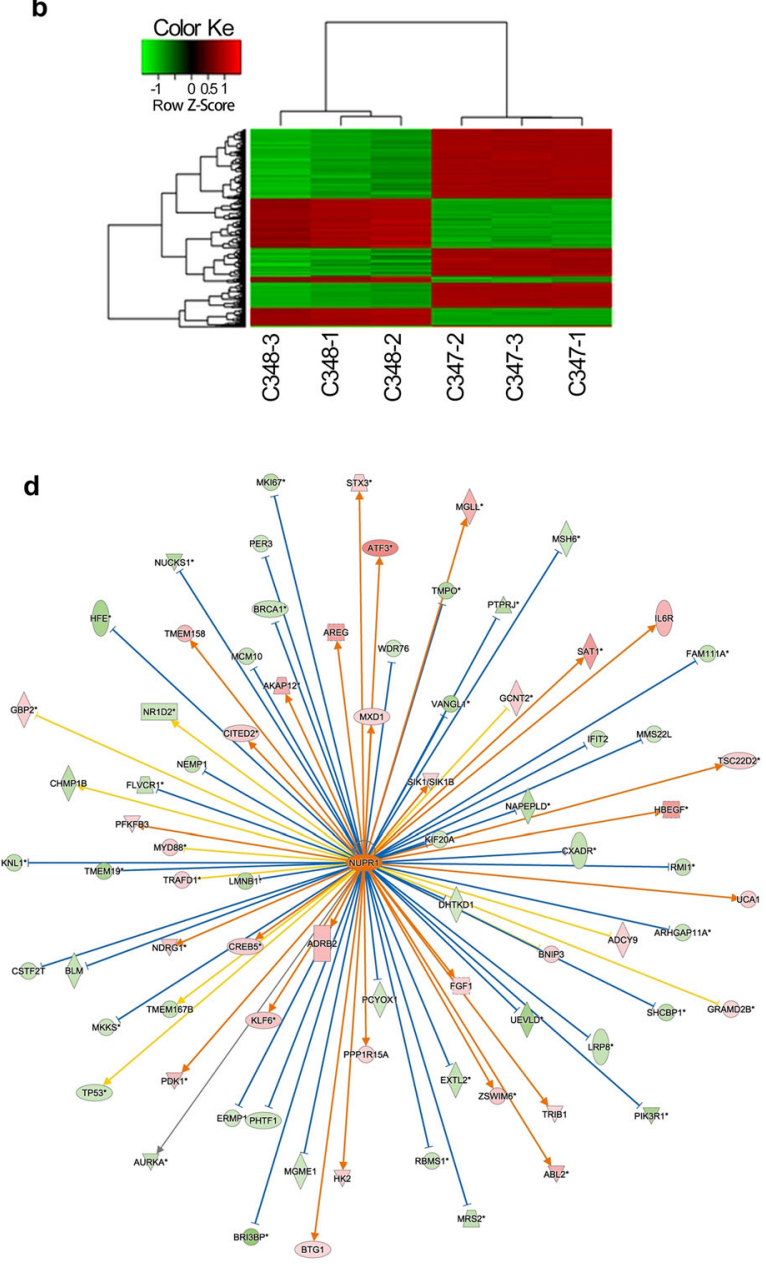

Fig. 7 LANCL2 knockdown enriched cancer-related signaling pathways and proteins. a Volcano plot analysis of gene chip array results revealed 496 upregulated and 839 downregulated DEGs after LANCL2 knockdown (DEG criteria: $p<0.05$; absolute fold-change $>2.0$ ). b Heatmap of DEGs after LANCL2 knockdown. Each row refers to one DEG. Each column represents one sample. The legend depicts the color code for Row Z-score foldchange values. c IPA interaction network analysis of DEGs detecting putative regulatory interactors of LANCL2. Note the highlighted putative interactions between LANCL2-EGFR, LANCL2-APP, and LANCL2-Akt. d IPA upstream regulator analysis of DEGs evidencing putative transcriptional regulators of LANCL2. e IPA downstream effects analysis of DEGs identifying putative dysregulated biological functions.

LANCL2 protein interactors in PC9 cells using Co-IP/MS analysis (Supplementary Fig. S11a). Seven proteins (FLNA, GSTM3, FASN, PKLR, TRIM25, YWHAB, and YWHAE) were identified as LANCL2 protein interactor candidates, based on the selection of Co-IP/MS proteins with an established relationship to cell proliferation
(Supplementary Fig. S11b). Follow-up Co-IP immunoblotting demonstrated that LANCL2 interacts with FLNA and GSTM3 (Supplementary Fig. S11c). Notably, these two proteins have not been previously identified as LANCL2 protein interactors by STRING analysis (Supplementary Fig. S11d). 


\section{Discussion}

EGFR is a transmembrane tyrosine kinase receptor and key activator of intracellular Akt signaling via PI3K ${ }^{9,30}$. Akt hyperactivity has been evidenced to promote EGFRmutant LUAD cell proliferation and chemoresistance ${ }^{31}$. LANCL2 plays a key role in promoting Akt hyperactivity in response to mitogenic signaling ${ }^{20}$; however, its involvement in EGFR TKI resistance in EGFR-mutant LUAD is largely uncharacterized. Herein, we evidenced that a positive correlation exists between LANCL2 and EGFR in LUAD patients, and LANCL2 gain-of-function is associated with poor survival in these patients. We showed that the EGFR-mutant LUAD cell lines PC9 and HCC827 displayed higher LANCL2 expression than the nonEGFR-mutant cell line A549. We also found that LANCL2 was downregulated following gefitinib+pemetrexed combination therapy in PC9 cells. Additionally, the shRNA-mediated knockdown of LANCL2 reduced proliferation and enhanced apoptosis in the EGFR-mutant LUAD cell lines PC9 and HCC827 in vitro and suppressed PC9 xenograft tumor growth in vivo. Moreover, we discovered that LANCL2 overexpression rescued these effects in LANCL2-silenced cells and promoted gefitinib +pemetrexed resistance in these EGFR-mutant LUAD cell lines. Furthermore, pathway analyses of DEGs from LANCL2 knockdown revealed significant enrichment for several cancer signaling pathways in PC9 cells. These combined results suggest that LANCL2 plays a key role in promoting EGFR-mutant LUAD.

In addition to its traditional tyrosine kinase function, EGFR signaling affects a number of tyrosine kinaseindependent pathways ${ }^{12,16,32}$. Both tyrosine kinasedependent and tyrosine kinase-independent EGFR signaling are able to hyperactivate pro-proliferative PI3K/ Akt pathways ${ }^{10}$. Gefitinib-resistant cells do not respond to tyrosine kinase pathway inhibition, therefore targeting interactors in EGFR's tyrosine kinase-independent pathways, such as LANCL2, to suppress Akt hyperactivity and cell proliferation could provide a useful strategy for bypassing EGFR TKI resistance in EGFR-mutant LUAD tumors. Interestingly, IPA analysis of the gene chip array data from LANCL2-knockdown PC9 cells evidenced that LANCL2 putatively interacts with EGFR as well as its traditional target Akt. Our follow-up qPCR analysis demonstrated that LANCL2 knockdown paradoxically promotes EGFR and APP mRNA expression levels in PC9 cells. APP is a putative protein interactor of EGFR ${ }^{33}$, and APP phosphorylation has been associated with poor survival in LUAD patients ${ }^{34}$. These findings may represent a regulatory-feedback mechanism to increase tumorigenic EGFR-Akt signaling in cells with LANCL2 knockdowninduced deficits in Akt activity. Therefore, this putative LANCL2-APP-EGFR-Akt regulatory sub-network should be further investigated.
Co-IP/MS analysis identified two novel protein interactors for LANCL2, namely FLNA and GSTM3, which may influence pro-proliferative effects of LANCL2 in EGFR-mutant LUAD cells. Filamin A (FLNA) is a cytoskeletal scaffolding protein that plays a key role in cellular signal transduction and is aberrantly expressed in several cancers $^{33}$. Silencing FLNA expression in A549 EGFR-WT LUAD cells activates EGFR signaling as well as cell proliferation, migration, and invasiveness in vitro. This suggests that FLNA is a negative regulator of EGFR activation in LUAD cells ${ }^{33}$. We speculate that binding between LANCL2 and FLNA may serve to negatively regulate FLNA activity, thereby promoting EGFR-Akt activity in EGFR-mutant LUAD cells. GSTM3 is a member of the glutathione S-transferase family of enzymes and participates in a wide variety of cellular functions, including the inhibition of apoptosis and detoxification of xenobiotics ${ }^{35}$. The dysregulation of GSTM3 expression is exhibited in several human malignancies, including lung, colorectal, prostate, and triple-negative breast cancer ${ }^{36-39}$. Although early research suggested a weak association exists between certain GST gene mutations (e.g., GSTM1, GSTM3, GSTP1, and GSTT1) and risk of lung cancer ${ }^{40,41}$, more recent work has not evidenced any definitive links between GSTM3 polymorphisms and lung cancer risk $^{42,43}$. However, molecular research in cervical cancer cells has revealed that GSTM3 upregulation is associated with promoting cancer cell survival, proliferation, tumor progression, and aberrant nuclear factor kappa B (NF- $\mathrm{kB}$ ) and mitogen-activated protein kinase signaling ${ }^{44}$; therefore, further research is required to clarify whether GSTM3 plays a role in EGFR-mutant LUAD.

It should be noted that LANCL2's effects on proliferation and apoptosis were not unique to the EGFRmutant LUAD cell lines, as the non-EGFR-mutant LUAD cell line A549 was also significantly affected by LANCL2 knockdown. This observation may be attributable to the high endogenous EGFR activity displayed by A549 cells relative to other LUAD cell lines ${ }^{45}$. Moreover, as gefitinib+pemetrexed combination therapy downregulates LANCL2 expression in PC9 cells, it may also produce a similar effect in non-EGFR-mutant LUAD cells. Further research on a panel of non-EGFRmutant LUAD cell lines with differing levels of endogenous EGFR activity and LANCL2 expression is needed to address these issues.

\section{Conclusion}

LANCL2 promotes tumorigenic proliferation, suppresses apoptosis, and promotes gefitinib + pemetrexed resistance in EGFR-mutant LUAD cells. Based on the positive association between LANCL2, EGFR, and downstream Akt signaling, LANCL2 may be a promising new therapeutic target for EGFR-mutant LUAD. 


\section{Funding}

The author(s) disclose receipt of the following financial support for the research, authorship, and/or publication of this article. This research was supported by the National Natural Science Foundation of China [grant number 81874037], the Key Projects of the Biomedicine Department, Science and Technology Commission of Shanghai Municipality [grant number 11411951200], Shanghai Chest Hospital Project of Collaborative Innovation [grant number YJXT20190102] and Nurture Project of Basic Research of Shanghai Chest Hospital [grant number 2020YNJCM06]. The funders had no role in study design, data collection and analysis, decision to publish, or preparation of the manuscript

\section{Author contributions}

L.Y., X.J., Z.Y., and Z.W. wrote the manuscript. L.Y., X.J., Z.Y., Z.W., Z.X., G.P., and Z.H., performed the experiments. W.H., L.J., and H.B. conceived and designed the study; reviewed and revised the manuscript.

\section{Availability of data and material}

The data that support the findings of this study are available from the corresponding author upon reasonable request.

\section{Conflict of interest}

The authors declare no competing interests.

\section{Ethics statement}

All in vitro and in vivo experiments were approved in advance by the Institutional Review Board of the Shanghai Chest Hospital.

\section{Publisher's note}

Springer Nature remains neutral with regard to jurisdictional claims in published maps and institutional affiliations.

Supplementary information The online version contains supplementary material available at https://doi.org/10.1038/s41419-021-03439-8.

Received: 11 August 2020 Revised: 13 January 2021 Accepted: 15 January 2021

Published online: 10 February 2021

\section{References}

1. Torre, L. A. et al. Global cancer statistics, 2012. CA Cancer J. Clin. 65, 87-108 (2015).

2. Lee, D. H. Treatments for EGFR-mutant non-small cell lung cancer (NSCLC): the road to a success, paved with failures. Pharm. Ther. 174, 1-21 (2017).

3. Metro, G. \& Crinò, L. Advances on EGFR mutation for lung cancer. Transl. Lung Cancer Res. 1, 5-13 (2012)

4. Yang, J. C.-H. et al. A review of regimens combining pemetrexed with an epidermal growth factor receptor tyrosine kinase inhibitor in the treatment of advanced nonsquamous non-small-cell lung cancer. Clin. Lung Cancer 19, 27-34 (2018)

5. Chong, C. R. \& Jänne, P. A. The quest to overcome resistance to EGFR-targeted therapies in cancer. Nat. Med. 19, 1389-1400 (2013).

6. Sequist, L. V. et al. Genotypic and histological evolution of lung cancers acquiring resistance to EGFR inhibitors. Sci. Transl. Med. 3, 75 ra26 (2011)

7. Helena, A. Y. et al. Analysis of tumor specimens at the time of acquired resistance to EGFR-TKI therapy in 155 patients with EGFR-mutant lung cancers. Clin. Cancer Res. 19, 2240-2247 (2013).

8. Greenberg, A. S. et al. The role of lipid droplets in metabolic disease in rodents and humans. J. Clin. Invest. 121, 2102-2110 (2011).

9. Huang, L. \& Fu, L. Mechanisms of resistance to EGFR tyrosine kinase inhibitors. Acta Pharm. Sin. B 5, 390-401 (2015).

10. Cully, M., You, H., Levine, A. J. \& Mak, T. W. Beyond PTEN mutations: the PI3K pathway as an integrator of multiple inputs during tumorigenesis. Nat. Rev. Cancer 6, 184-192 (2006).
11. Chen, G., Kronenberger, P., Teugels, E., Umelo, I. A. \& De Grève, J. Targeting the epidermal growth factor receptor in non-small cell lung cancer cells: the effect of combining RNA interference with tyrosine kinase inhibitors or cetuximab. BMC Med. 10, 28 (2012).

12. Ewald, J. A., Wilkinson, J. C., Guyer, C. A. \& Staros, J. V. Ligand-and kinase activity-independent cell survival mediated by the epidermal growth factor receptor expressed in 32D cells. Exp. Cell Res. 282, 121-131 (2003).

13. Feng, F. et al. Role of epidermal growth factor receptor degradation in gemcitabine-mediated cytotoxicity. Oncogene 26, 3431-3439 (2007).

14. Nagy, P., Arndt-Jovin, D. J. \& Jovin, T. M. Small interfering RNAs suppress the expression of endogenous and GFP-fused epidermal growth factor receptor (erbB1) and induce apoptosis in erbB1-overexpressing cells. Exp. Cell Res. 285, 39-49 (2003).

15. Tan, X., Thapa, N., Sun, Y. \& Anderson, R. A. A kinase-independent role for EGF receptor in autophagy initiation. Cell 160, 145-160 (2015).

16. Weihua, Z. et al. Survival of cancer cells is maintained by EGFR independent of its kinase activity. Cancer Cell 13, 385-393 (2008).

17. Lawlor, M. A. \& Alessi, D. R. PKB/Akt: a key mediator of cell proliferation, survival and insulin responses? J. Cell Sci. 114, 2903-2910 (2001).

18. Bellacosa, A., Kumar, C. C., Di Cristofano, A. \& Testa, J. R. Activation of AKT kinases in cancer: implications for therapeutic targeting. Adv. Cancer Res. 94 29-86 (2005).

19. Franke, T. F. Intracellular signaling by Akt: bound to be specific. Sci. Signal 1, pe29 (2008).

20. Zeng, M., Van Der Donk, W. A. \& Chen, J. Lanthionine synthetase C-like protein 2 (LanCL2) is a novel regulator of Akt. Mol. Biol. Cell $\mathbf{2 5}$ 3954-3961 (2014)

21. Cerami, E. et al. The cBio cancer genomics portal: an open platform for exploring multidimensional cancer genomics data. Cancer Discov. 2, 401-404 (2012).

22. Chandrashekar, D. S. et al. UALCAN: a portal for facilitating tumor subgroup gene expression and survival analyses. Neoplasia 19 649-658 (2017).

23. La Monica, S. et al. Combination of gefitinib and pemetrexed prevents the acquisition of TKI resistance in NSCLC cell lines carrying EGFR-activating mutation. J. Thorac. Oncol. 11, 1051-1063 (2016).

24. Jia, P. et al. Next-generation sequencing of paired tyrosine kinase inhibitorsensitive and-resistant EGFR mutant lung cancer cell lines identifies spectrum of DNA changes associated with drug resistance. Genome Res. 23, 1434-1445 (2013).

25. Chabon, J. J. et al. Circulating tumour DNA profiling reveals heterogeneity of EGFR inhibitor resistance mechanisms in lung cancer patients. Nat. Commun. 7, 1-15 (2016).

26. Li, H. et al. Decreased glutathione biosynthesis contributes to EGFR T790Mdriven erlotinib resistance in non-small cell lung cancer. Cell Discov. 2, 1-14 (2016).

27. Bindea, G. et al. ClueGO: a Cytoscape plug-in to decipher functionally grouped gene ontology and pathway annotation networks. Bioinformatics $\mathbf{2 5}$ 1091-1093 (2009).

28. Krämer, A., Green, J., Pollard, J. Jr. \& Tugendreich, S. Causal analysis approaches in ingenuity pathway analysis. Bioinformatics 30, 523-530 (2013).

29. Szklarczyk, D. et al. STRING v11: protein-protein association networks with increased coverage, supporting functional discovery in genome-wide experimental datasets. Nucleic Acids Res. 47, D607-D613 (2018).

30. Murillo, M. M., Rana, S, Spencer-Dene, B, Nye, E, Stamp, G. \& Downward, J. Disruption of the Interaction of RAS with PI 3-kinase induces regression of EGFR-mutant-driven lung cancer. Cell Rep. 25, 3545-3553 (2018).

31. Rotow, J. \& Bivona, T. G. Understanding and targeting resistance mechanisms in NSCLC. Nat. Rev. Cancer 17, 637-658 (2017).

32. Huber, S. M., Misovic, M., Mayer, C., Rodemann, H.P. \& Dittmann, K. EGFRmediated stimulation of sodium/glucose cotransport promotes survival of irradiated human A549 lung adenocarcinoma cells. Radiother. Oncol. 103, 373-379 (2012).

33. Zhang, H., Liu, J., Fu, X. \& Yang, A. Identification of key genes and pathways in tongue squamous cell carcinoma using bioinformatics analysis. Med Sci. Monit. 23, 5924-5932 (2017).

34. Ito, S. et al. Amyloid precursor protein and its phosphorylated form in nonsmall cell lung carcinoma. Pathol. Res Pract. 215, 152463 (2019).

35. Hayes, J. D., Flanagan, J. U. \& Jowsey, I. R. Glutathione transferases. Annu. Rev. Pharm. Toxicol. 45, 51-88 (2005). 
36. Meding, S. et al. Tissue-based proteomics reveals FXYD3, S100A11 and GSTM3 as novel markers for regional lymph node metastasis in colon cancer. J. Pathol. 228, 459-470 (2012)

37. Medeiros, R. et al. Metabolic susceptibility genes and prostate cancer risk in a southern European population: the role of glutathione Stransferases GSTM1, GSTM3, and GST11 genetic polymorphisms. Prostate 58, 414-420 (2004).

38. Louie, S. M. et al. GSTP1 is a driver of triple-negative breast cancer cell metabolism and pathogenicity. Cell Chem. Biol. 23, 567-578 (2016).

39. Loktionov, A. et al. Glutathione-S-transferase gene polymorphisms in colorectal cancer patients: interaction between GSTM1 and GSTM3 allele variants as a risk-modulating factor. Carcinogenesis 22, 1053-1060 (2001).

40. Saarikoski, S. T. et al. Combined effect of polymorphic GST genes on individual susceptibility to lung cancer. Int. J. Cancer 77, 516-521 (1998).
41. Jourenkova-Mironova, N. et al. Role of glutathione S-transferase GSTM1, GSTM3, GSTP1 and GSTT1 genotypes in modulating susceptibility to smokingrelated lung cancer. Pharmacogenetics 8, 495-502 (1998).

42. Ye, Z., Song, H., Higgins, J. P., Pharoah, P. \& Danesh, J. Five glutathione stransferase gene variants in 23,452 cases of lung cancer and 30,397 controls: meta-analysis of 130 studies. PLoS Med. 3, e91 (2006).

43. Feng, $X$. et al. Lack of association of glutathione S-transferase M3 gene polymorphism with the susceptibility of lung cancer. Asian Pac. J. Cancer Prev. 13, 4465-4468 (2012).

44. Checa-Rojas, A. et al. GSTM3 and GSTP1: novel players driving tumor progression in cervical cancer. Oncotarget 9, 21696-21714 (2018).

45. Van Schaeybroeck, S. et al. Chemotherapy-induced epidermal growth factor receptor activation determines response to combined gefitinib/chemotherapy treatment in non-small cell lung cancer cells. Mol. Cancer Ther. 5 1154-1165 (2006) 